\title{
Uric Acid Nephrolithiasis
}

National Cancer Institute

\section{Source}

National Cancer Institute. Uric Acid Nephrolithiasis. NCI Thesaurus. Code C123037.

Nephrolithiasis in which the composition of the stone(s) is predominantly uric acid. 